\title{
Mental health: a dental perspective
}

\author{
By Faisal Moughal, 26, Associate in mixed practice
}

$\mathrm{B}$ eing a recently qualified graduate, I intended to get a wide range of experiences. I was able to get exposure to different niches within the profession, with a year in hospital followed by work in both mixed and private practice. An opportunity arose for me to become the GDP at a charity run specialist mental health facility, taking on vulnerable patients with complex and challenging needs. The demographic treated represented those with severe mental health afflictions, with an age range broadly from adolescent upwards. I gained an insight into the different circumstances and associated challenges in the provision of care away from normal practice. Considering the wider needs of this particular group of patients, approaching the treatment from a holistic viewpoint was especially important, with the focus certainly on prevention and stabilisation.

The differences in the working environment were stark, with most patients escorted by healthcare assistants, and longer times allotted to the appointments.

These patients have such extreme challenges elsewhere that the mouth takes a back seat and building trust takes much longer. Similar to practice, I believed an evidence based ${ }^{1}$ approach of regular reinforcement of prevention would improve the success of any future more complex treatment.

A big obstacle faced was that of unreasonable treatment requests by patients, often due to a lack of insight stemming from their mental health conditions. Ultimately the Mental Capacity $\mathrm{Act}^{2}$ states a person mustn't be treated as unable to make a decision just because it is unwise. However, this is balanced against the need of the clinician to proceed only in the best interest of patients. One example is of a patient who wanted gold crowns across their anterior dentition despite gross caries elsewhere; obviously this wasn't appropriate so an approach of reasoning and explanation was taken, involving those the patient was close to with their permission, and eventually a more suitable plan of stabilisation was agreed upon.
Considerations surrounding informed consent were especially important here, with the guidance stating a person is assumed to have capacity unless established otherwise, and help and guidance should be given to enable them to make their own decisions. Ultimately with this particular cohort of patients often a decision had been made regarding their capacity to consent to mental health treatments, but this didn't necessarily always translate to their capacity surrounding dental procedures. It was clear capacity was fluid, and through extensive, repeated discussions patients were able to make decisions regarding their dental needs, and every effort was made to enable this. At each appointment the treatment plan was reinforced, patients were given time to discuss their needs with those close to them, and oral health education sessions were carried out alongside their general educational sessions. Regular liaison with their responsible clinicians was extremely valuable, allowing a well informed and rounded decision to be made. In the rare cases where a patient was not deemed to have capacity, the assistance of their responsible clinician in coming to a decision on what treatment is necessary and least restrictive was used.

Throughout my time I came across a range of colourful personalities, and getting to know and support patients with such complex challenges was the most rewarding part of the role. The experiences allowed me to develop my abilities in both risk assessment and management of expectations, skills transferable to work in different environments. Being able to help those with such significant afflictions was very rewarding, and I look forward to putting the skills gained to use in my future career.

\section{References}

1. Public Health England. Delivering better oral health. Third edition, 2014, Available at: https://assets. publishing.service.gov.uk/government/uploads/system/uploads/attachment_data/file/605266/Delivering_better_oral_health.pdf (accessed March 2020).

2. Parliament of the UK. Mental Capacity Act 2005. Available at: http://www.legislation.gov. uk/ukpga/2005/9/contents (accessed March 2020).

\section{BDA begins resolving associate pay disputes}

The British Dental Association (BDA) has launched an associate member pay dispute resolution service. It is working to ensure that associates who deliver NHS treatments (whether exclusively or in a mixed practice) received some financial security during the COVID-19 pandemic.

Following successful lobbying, corporate bodies and practice owners with NHS contracts will continue to be paid assuming certain conditions are met. One of those is that they are obliged to pass on income due to associates. Practices are also required to support the wider NHS pandemic effort, and this is likely to involve associates if NHS money is to be passed on.

The pay dispute service's primary aim is to ensure that any issues are resolved in a fair and satisfactory way for everyone involved so that effective working relationships are maintained.

New cases can be logged at https://www.bda.org/advice/ Coronavirus/Pages/associates-coronavirus.aspx.

Chair of the BDA's Associate Group Sarah Canavan said: 'The overwhelming majority of corporates and practice owners are acting in line with the deals we've struck with governments. But in those rare cases where this isn't happening we are already working to resolve matters swiftly and constructively.

'We are setting out to help all parties reach a fair agreement in line with NHS rules. At this difficult time, we are offering associates potentially the only route they have for real clarity on their pay.' 